WU B 13-16

October, 242013

\title{
Probing moments of baryon-antibaryon generalized parton distributions at BELLE and FAIR
}

\author{
P. Kroll ${ }^{a b}{ }^{1}$ and A. Schäfer ${ }^{a}$ \\ a: Institut für Theoretische Physik, Universität Regensburg, D-93040, Germany \\ b: Fachbereich Physik, Universität Wuppertal, D-42097 Wuppertal, Germany
}

\begin{abstract}
We analyze the time-like processes $\gamma \gamma \rightarrow B \bar{B}$ and $p \bar{p} \rightarrow \gamma M$ at large Mandelstam variables within the handbag approach for which the process amplitudes factorize in hard partonic subprocesses and annihilation form factor. The latter represent moments of baryonantibaryon generalized parton distributions (GPDs). Symmetry relations restrict the number of independent annihilation form factors for the ground state baryons drastically. We determine these form factors from the present BELLE data on $\gamma \gamma \rightarrow B \bar{B}$ with the help of simplifying assumptions. The knowledge of these form factors allow for predictions of $p \bar{p} \rightarrow \gamma M$ for various mesons which may be probed at FAIR.
\end{abstract}

\section{Introduction}

For nearly twenty years hard exclusive reactions attracted much attention from both Theoreticians and Experimentalists. This in particular true for the deeply virtual processes, Compton scattering and meson leptoproduction,

\footnotetext{
${ }^{1}$ Email: kroll@physik.uni-wuppertal.de

${ }^{2}$ Email: andreas.schaefer@physik.uniregensburg.de
} 
where factorization theorems tell us that, in the generalized Bjorken regime of large photon virtuality and large energy, a process amplitude can be expressed as a convolution of hard, perturbatively calculable partonic subprocess amplitudes and soft hadronic matrix elements, parametrized as GPDs. In the course of time the accumulated data revealed the consistency of the theoretical concept, the so-called handbag approach, even though there are phenomena in the experimental data which seem to indicate the existence of strong corrections to the asymptotic factorization formula. It has been argued [1] that these phenomena can, fully or partially, be accounted for by particular choices of the GPDs, i.e. they can be described by logarithms of the photon virtuality instead of powers as advocated for in [2]. Whether or not this is possible for most or all these phenomena is yet unsolved. Deeply virtual exclusive processes have also been studied in the time-like region, e.g. $\gamma^{*} \gamma \rightarrow \pi^{+} \pi^{-}[3]$ or $p \bar{p} \rightarrow \gamma^{*} \pi$ [4]. Lack of data prevents the verification of this factorization approach till now.

Complementary to the deeply virtual reaction are the exclusive wide-angle processes as for instance real Compton scattering $[5,6]$ or their time-like counter part, the annihilation of two real photons into a pair of hadrons [7][10]. The hard scale which permits factorization into hard and soft physics in this case, is provided by large Mandelstam variables $s \sim-t \sim-u$. The amplitudes are then expressed as a product of hard subprocess, $\gamma q \rightarrow \gamma q$ or $\gamma \gamma \rightarrow q \bar{q}$, and form factors representing moments of GPDs. In contrast to the deeply virtual reactions where factorization has rigorously been proven, factorization of the wide-angle reactions can only be shown to hold with the help of assumptions. Thus, for two-photon annihilation into a baryon-antibaryon pair factorization of the process amplitudes is achieved with the plausible assumption [8] that the process is dominated by configurations where the quark created in the subprocess, approximately moves in the direction of the baryon, whereas the antiquark moves in the direction of the antibaryon. This assumption is equivalent to the familiar valence quark approximation. By time reversal the amplitudes for two-photon annihilation into a protonantiproton pair are the same as those for $p \bar{p} \rightarrow \gamma \gamma$ (up to eventual signs). As shown in [11] the latter process can be generalized by replacing one of the photons in the final state by a meson.

In this work we provide an update of [8] taking into account the new BELLE data for $\gamma \gamma \rightarrow B \bar{B}[12,13]$ (where $B$ is a ground-state baryon). We now include the annihilation form factor for non-valence quarks in the numerical analysis as turned out to be necessary for $\gamma \gamma$ annihilations into 
a pair of pseudoscalar mesons [10]. In contrast to our previous work [11] we are now going to explore in some detail the process $p \bar{p} \rightarrow \gamma V_{L}$ where $V_{L}$ is a longitudinally polarized vector meson. Both the classes of reactions, $\gamma \gamma \rightarrow B \bar{B}$ and $p \bar{p} \rightarrow \gamma V_{L}$, are linked to each other in so far as the same soft $B \bar{B}$ annihilation form factors occur. We think of our study as being timely since, in a few years from now, BELLE II and FAIR will be put into operation. In the first experiment the $\gamma \gamma \rightarrow B \bar{B}$ processes can be measured with high accuracy while at FAIR the proton-antiproton initiated reactions can be studied experimentally.

Plan of the paper is the following: In Sect. 2 we sketch the handbag approach to wide-angle time-like exclusive processes. The symmetry relations among the annihilation form factors and the phenomenological determination of the set of independent form factors are discussed in Sect. 3. The next section is devoted to applications of the handbag approach to protonantiproton annihilation into a photon and a meson and Sect. 5 to the special case of the $J / \Psi$. The paper ends with a summary and an outlook (Sect. 6).

\section{The handbag approach}

In $[8,9]$ it has been argued that for large Mandelstam variables $s,-t$ and $-u$, the amplitudes for two-photon annihilations into pairs of ground state baryons factorize in a hard subprocess, $\gamma \gamma \rightarrow q \bar{q}$, and in soft annihilation form factors, $R_{i}^{\gamma}(s)$. In this so-called handbag approach the differential cross section takes the form

$$
\frac{d \sigma}{d t}(\gamma \gamma \rightarrow B \bar{B})=\frac{4 \pi \alpha_{\mathrm{elm}}^{2}}{s^{2}} \frac{1}{\sin ^{2} \theta}\left[\left|R_{V}^{\gamma}(B \bar{B}, s)\right|^{2} \cos ^{2} \theta+\left|R_{\mathrm{eff}}^{\gamma}(B \bar{B}, s)\right|^{2}\right]
$$

where $\theta$ is the scattering angle in the center-of-mass system and the effective annihilation form factor is short for the combination $\left(m_{B}\right.$ is the mass of the baryon)

$$
\left|R_{\mathrm{eff}}^{\gamma}(s)\right|=\sqrt{\left|R_{A}^{\gamma}(s)+R_{P}^{\gamma}(s)\right|^{2}+\frac{s}{4 m_{B}^{2}}\left|R_{P}^{\gamma}(s)\right|^{2}} .
$$

The form factors represent the lowest moments of $B \bar{B}$ distribution amplitudes, $\Phi_{i}^{q}$

$$
F_{B \bar{B} i}^{q}(s)=\int_{0}^{1} d z \Phi_{B \bar{B} i}^{q}(z, \zeta, s)
$$


multiplied with the correspondent quark charges and summed over flavors $(q=u, d, s, i=V, A, P)$,

$$
R_{i}^{\gamma}(B \bar{B}, s)=e_{u}^{2} F_{B \bar{B} i}^{u}(s)+e_{d}^{2} F_{B \bar{B} i}^{d}(s)+e_{s}^{2} F_{B \bar{B} i}^{s}(s) .
$$

Here $e_{q}$ is the charge of the quark of flavor $q$ in units of the positron charge $e_{0}$ and ${ }^{3}$ The $B \bar{B}$ distribution amplitudes and hence their moments are universal, i.e. process independent while the annihilation form factors are processdependent flavor combinations of the moments $F_{B \bar{B}}^{q}$.

In the time-like region the skewness is defined by

$$
\zeta=\frac{p^{+}}{p^{+}+p^{\prime+}}
$$

where $p^{+}$and $p^{\prime+}$ are the light-cone plus components of the baryon and antibaryon momenta, respectively. The scattering-angle dependence in (1) comes from the hard subprocess which is computed to lowest order of QED. By time-reversal invariance (1) also holds for the process $B \bar{B} \rightarrow \gamma \gamma$.

As discussed in detail in $[7,8]$ the factorized cross section (1) is derived in a center-of-mass frame where the process takes place in the 1-3 plane and the outgoing hadrons move along the positive or negative 1-direction. In this frame both the hadron momenta have the same plus components and, hence, skewness is $1 / 2$. In principle there is also a scalar distribution amplitude and a corresponding scalar form factor. However, in a $\zeta=1 / 2$ frame the scalar form factor decouples [8].

The $q \bar{q} \rightarrow B \bar{B}$ transitions and therefore the annihilation form factors can only be soft if the quark and the antiquark have small virtualities and momenta that are approximately equal to those of the baryon and antibaryon $(z \simeq 1 / 2)$, respectively. Corrections to this approximations are of order $\Lambda^{2} / s$ where $\Lambda$ is a typical hadronic scale of order $1 \mathrm{GeV}$. Thus, the dynamics we consider can be viewed as a time-like version of the Feynman mechanism.

The handbag approach to $\gamma \gamma \rightarrow B \bar{B}$ or better $B \bar{B} \rightarrow \gamma \gamma$ can straightforwardly be generalized to processes for which one of the photons is replaced by pseudoscalar $(P)$ or a longitudinally polarized vector meson $\left(V_{L}\right)$ [11]. Of experimental interest are the processes with ingoing proton and antiproton

$$
\frac{d \sigma}{d t}(p \bar{p} \rightarrow \gamma P)=\frac{\alpha_{\mathrm{elm}}}{2 s^{3}} \frac{\left|\bar{a}_{P}\right|^{2}}{\sin ^{4} \theta}\left[\left|R_{\mathrm{eff}}^{P}(s)\right|^{2}+\cos ^{2} \theta\left|R_{V}^{P}(s)\right|^{2}\right],
$$

\footnotetext{
${ }^{3}$ The exact definition of the $B \bar{B}$ distribution amplitudes, the time-like versions of generalized parton distributions, can be found in [8]. The scale dependence of the distribution amplitudes is not displayed for convenience.
} 


$$
\frac{d \sigma}{d t}\left(p \bar{p} \rightarrow \gamma V_{L}\right)=\frac{\alpha_{\mathrm{elm}}}{2 s^{3}} \frac{\left|\bar{a}_{V}\right|^{2}}{\sin ^{4} \theta}\left[\cos ^{2} \theta\left|R_{\mathrm{eff}}^{V_{L}}(s)\right|^{2}+\left|R_{V}^{V_{L}}(s)\right|^{2}\right]
$$

Since only the proton-antiproton initial state is experimentally feasible we omit the label $p \bar{p}$ of the form factors for the ease of reading. The form factor $R_{\text {eff }}^{M}$ denotes the combination of $R_{A}^{M}$ and $R_{P}^{M}$ analogously to (2). The hard subprocess $q \bar{q} \rightarrow \gamma M$ is to be calculated perturbatively from the onegluon exchange contribution of which a typical Feynman graph is shown in Fig. 1. In this dynamical mechanism the meson is generated from its valence Fock component in collinear approximation. Consequently, the meson selects its valence (anti)quarks from the (anti)proton. In terms of the universal moments of the generalized distribution amplitudes the annihilation form factors read

$$
\begin{aligned}
R_{i}^{\rho^{0}, \pi^{0}} & =\frac{1}{\sqrt{2}}\left(e_{u} F_{i}^{u}-e_{d} F_{i}^{d}\right), \\
R_{i}^{\omega_{q}, \eta_{q}} & =\frac{1}{\sqrt{2}}\left(e_{u} F_{i}^{u}+e_{d} F_{i}^{d}\right), \\
R_{i}^{\omega_{s}, \eta_{s}} & =e_{s} F_{i}^{s} .
\end{aligned}
$$

The $\eta_{q}\left(\omega_{q}\right)$ and $\eta_{s}\left(\omega_{s}\right)$ are the non-strange $((u \bar{u}+d \bar{d}) / \sqrt{2})$ and strange $(s \bar{s})$ components of the physical $\eta(\omega)$ and $\eta^{\prime}(\phi)$ mesons [14] which are the basis states in the quark-flavor basis. The mixing angle, $\Phi_{P}$, of the pseudoscalars in this basis amounts to $39.3^{\circ}[14,15]$. For the $\omega$ and $\phi$ system the corresponding mixing angle, $\Phi_{V}$, is known to be very small: from the $\phi \rightarrow \pi^{0} \gamma$ and $\omega \rightarrow \pi^{0} \gamma$ branching ratios [16] one finds a value of $3.3^{\circ}$ for the vector-meson mixing angle [17]. For the vector-meson channels there is in principle also a contribution from the gluonic subprocess $g g \rightarrow \gamma V_{L}$ along with the gluonic form factors $F_{i}^{g}(s)$. However, this contribution is zero as has been note earlier in [18] for the space-like process $\gamma p \rightarrow V_{L} p$ and in [19] for $\gamma g \rightarrow J / \psi g$ in the limit $M_{J / \psi} \rightarrow 0$.

Evaluating the function $\bar{a}_{M}$ to leading order of perturbation theory, one finds

$$
\bar{a}_{M}^{\text {coll }}=4 \pi \alpha_{\mathrm{s}}\left(\mu_{R}\right) \frac{C_{F}}{N_{c}} f_{M}\langle 1 / \tau\rangle_{M}
$$

in collinear approximation where $f_{M}$ is the meson's decay constant, $\langle 1 / \tau\rangle_{M}$ is the $1 / \tau$ moment of its distribution amplitude $(\tau$ is the momentum fraction the quark entering the meson carries) and $\mu_{R}$ is an appropriate renormalization scale. The number of colors is denoted by $N_{c}$ and $C_{F}=\left(N_{c}^{2}-1\right) /\left(2 N_{c}\right)$. 

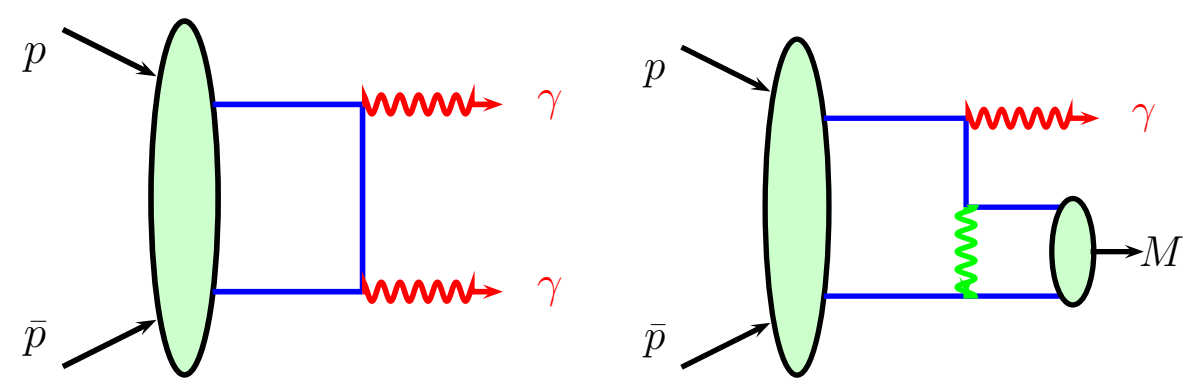

Figure 1: A sample Feynman graphs for $p \bar{p} \rightarrow \gamma \gamma$ and $p \bar{p} \rightarrow \gamma M$.

The $s-t$ crossed version of (6) is the handbag result for wide-angle meson photoproduction as derived in [18].

\section{Determination of the annihilation form fac- tors}

The handbag mechanism only involves quark-antiquark intermediate states, $\gamma \gamma \rightarrow q \bar{q} \rightarrow B \bar{B}$. Hence, isospin 2 (as well as $V$-spin 2) transitions are absent. Similar to the case of $\gamma \gamma \rightarrow M \bar{M}[7,10]$ this dynamical selection rule in combination with $\mathrm{SU}(3)$ flavor symmetry, in particular $U$-spin invariance, leads to relations among the moments $F_{B \bar{B}}^{q}$. For each $i=V, A, P$ there are only three independent form factors [8] for which we choose

$$
F_{i}^{q}=F_{p \bar{p} i}^{q} .
$$

In terms of these independent moments or basic form factors the annihilation form factors for the $\gamma \gamma \rightarrow B \bar{B}$ processes read

$$
\begin{aligned}
R_{i}^{\gamma}(p \bar{p}) & =e_{u}^{2} F_{i}^{u}+e_{d}^{2} F_{i}^{d}+e_{s}^{2} F_{i}^{s}, \\
R_{i}^{\gamma}(n \bar{n}) & =e_{u}^{2} F_{i}^{d}+e_{d}^{2} F_{i}^{u}+e_{s}^{2} F_{i}^{s}, \\
R_{i}^{\gamma}\left(\Sigma^{+} \bar{\Sigma}^{-}\right) & =e_{u}^{2} F_{i}^{u}+e_{d}^{2} F_{i}^{s}+e_{s}^{2} F_{i}^{d}, \\
R_{i}^{\gamma}\left(\Sigma^{-} \bar{\Sigma}^{+}\right) & =e_{u}^{2} F_{i}^{s}+e_{d}^{2} F_{i}^{u}+e_{s}^{2} F_{i}^{d}, \\
R_{i}^{\gamma}\left(\Sigma^{0} \bar{\Sigma}^{0}\right) & =-\frac{1}{2}\left(e_{u}^{2}+e_{d}^{2}\right)\left(F_{i}^{u}+F_{i}^{s}\right)-e_{s}^{2} F_{i}^{d}, \\
R_{i}^{\gamma}(\Lambda \bar{\Lambda}) & =-\frac{1}{6}\left(e_{u}^{2}+e_{d}^{2}\right)\left(F_{i}^{u}+4 F_{i}^{d}+F_{i}^{s}\right)-\frac{e_{s}^{2}}{3}\left(2 F_{i}^{u}-F_{i}^{d}+2 F_{i}^{s}\right),
\end{aligned}
$$




$$
R_{i}^{\gamma}\left(\Lambda \bar{\Sigma}^{0}\right)=R_{i}^{\gamma}\left(\Sigma^{0} \bar{\Lambda}\right)=-\frac{\sqrt{3}}{6}\left(e_{u}^{2}-e_{d}^{2}\right)\left(F_{i}^{u}-2 F_{i}^{d}+F_{i}^{s}\right)
$$

up to corrections due to breaking of flavor symmetry which we ignore in this work. The results for the form factors of the cascade particles for which flavor symmetry breaking is expected to be sizable, can be found in [8].

In principle it is possible to extract the absolute magnitude of the moments, $F_{i}^{q}$, as well as their relative phases from a sufficiently large set of data on $\gamma \gamma \rightarrow B \bar{B}$. From the angular dependence of the cross sections one may separate the effective form factors from the vector ones by a Rosenbluth type of separation. Measurements of the helicity correlation between the baryon and antibaryon would further allow to isolate $F_{P}^{q}$ from $F_{A}^{q}$. With regard to the accuracy of the present data on $\gamma \gamma \rightarrow B \bar{B}$ this program is not feasible currently. In order to estimate the $F_{i}^{q}$ we therefore simplify by assuming

$$
F_{i}^{d}=\rho_{d} F_{i}^{u}, \quad F_{i}^{s}=\rho_{s} F_{i}^{u} .
$$

Due to the simplification of choosing $i$-independent factors $\rho_{d}$ and $\rho_{s}$ we can introduce a combination of moments $F_{i}^{q}$ analogously to (2) which is related to $R_{\text {eff }}^{\gamma}$ by

$$
F_{\text {eff }}^{u}=R_{\text {eff }}^{\gamma}(p \bar{p})\left[e_{u}^{2}+e_{d}^{2} \rho_{d}+e_{s}^{2} \rho_{s}\right]^{-1} .
$$

We fit the basic form factors, i.e. $R_{\text {eff }}^{\gamma},\left|R_{V}^{\gamma}\right|, \rho_{d}$ and $\rho_{s}$, to the available data. Relative phases between the basic form factor are ignored since the available data do not allow to fix them. The accurate BELLE data on the $\gamma \gamma \rightarrow p \bar{p}$ differential and integrated cross sections [12] determine $R_{\text {eff }}^{\gamma}(p \bar{p})$ and $R_{V}^{\gamma}(p \bar{p})$ while the cross section data, integrated over the wide-angle region $(|\cos \theta| \leq 0.6)$, for the processes $\gamma \gamma \rightarrow \Lambda \bar{\Lambda}, \Sigma^{0} \bar{\Sigma}^{0}$ from BELLE [13], L3 [20] and CLEO [21] fix in addition $\rho_{d}$ and $\rho_{s}$. Data are taken into account only for $s$ larger than $7 \mathrm{GeV}^{2}$ (and leaving out the region of the $\eta_{c}$ formation). For $s$ smaller than about $7 \mathrm{GeV}^{2}$ the $\gamma \gamma \rightarrow p \bar{p}$ differential cross section reveals a maximum at a scattering angle of $90^{\circ}$ [12] which is in conflict with the properties of the handbag dynamics. A best fit provides $\left(s_{0}=10.4 \mathrm{GeV}^{2}\right)$

$$
\begin{aligned}
s^{2} R_{\mathrm{eff}}^{\gamma} & =(3.12 \pm 0.3)\left(s / s_{0}\right)^{-1.10 \pm 0.10} \mathrm{GeV}^{4}, \\
s^{2}\left|R_{V}^{\gamma}\right| & =(8.82 \pm 0.8)\left(s / s_{0}\right)^{-1.10 \pm 0.10} \mathrm{GeV}^{4}, \\
\rho_{d} & =0.55 \pm 0.15 \\
\rho_{s} & =-(0.13 \pm 0.11) s_{0} / s .
\end{aligned}
$$



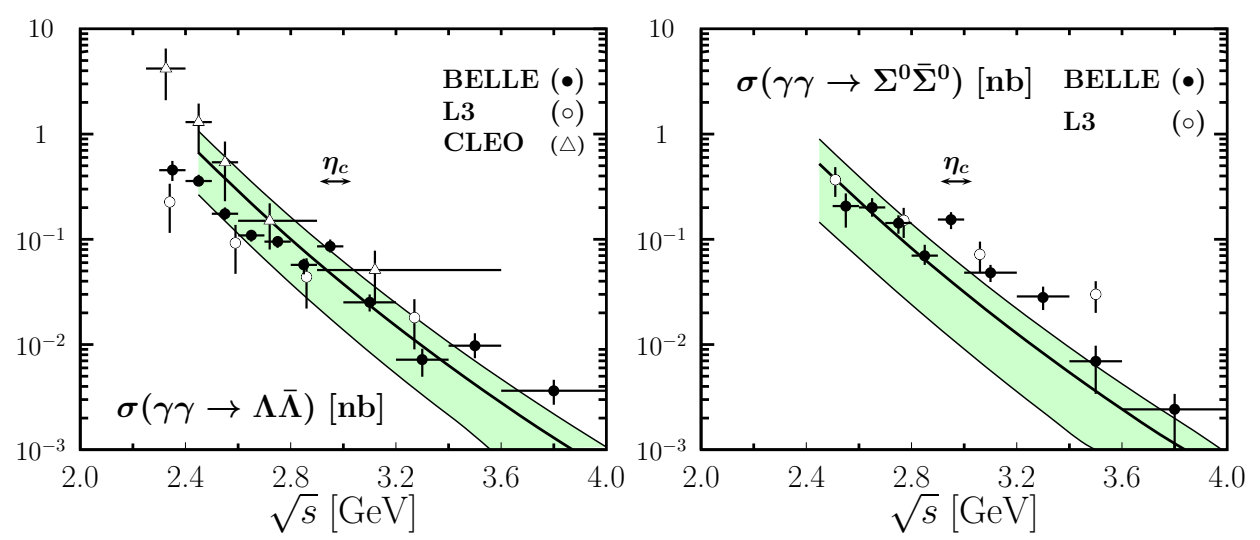

Figure 2: The integrated cross sections for $\gamma \gamma \rightarrow \Lambda \bar{\Lambda}$ (left) and $\gamma \gamma \rightarrow \Sigma^{0} \bar{\Sigma}^{0}$ (right) $(|\cos \theta|<0.6)$. Data are taken from BELLE [13] (preliminary), L3 [20] and CLEO [21]. The solid lines represent our fit, evaluated from (13), and the shaded bands its uncertainties.

Admittedly the fit is to be regarded as a rough estimate, the present data do not allow for a more accurate determination of the annihilation form factors. The fit to the $\gamma \gamma \rightarrow p \bar{p}$ differential and integrated cross section is practically the same as our previous fit presented in [11]. We therefore refrain from showing plots of the $\gamma \gamma \rightarrow p \bar{p}$ observables here. The fit to the $\Lambda \bar{\Lambda}$ and $\Sigma^{0} \bar{\Sigma}^{0}$ cross sections is compared to the data in Fig. 2. Note the sign of $\rho_{s}$ which is the same as the relative sign between the valence and non-valence form factors in the case of two-photon annihilations into pairs of mesons [10].

The cross sections for other $B \bar{B}$ channels can easily be worked out from (10) and (11). Their ratios to the $p \bar{p}$ cross section is fixed by quark charges and the parameters $\rho_{d}$ and $\rho_{s}$. Thus for instance, the ratio of the $n \bar{n}$ and $p \bar{p}$ cross sections is 0.49 at $s \simeq 30 \mathrm{GeV}^{2}$ and the $\Sigma^{-} \bar{\Sigma}^{+} / p \bar{p}$ ratio is 0.09 . The cross section for $\gamma \gamma \rightarrow \Lambda \bar{\Sigma}^{0}$ is very small, suppressed by about a factor 1000 as compared to the $p \bar{p}$ one. The non-valence form factor is very important in this case leading to a stronger energy dependence of that cross section than the other ones exhibit.

Before closing this section a remark is in order concerning an alternative mechanism for two-photon annihilation into a baryon-antibaryon pair. This is the so-called perturbative QCD factorization scheme [22, 23] which also holds for large Mandelstam variables. In this factorization scheme the valence 
Fock components of the proton and the antiproton are fully resolved in three quarks and antiquarks, respectively. Higher Fock components are suppressed by powers of the hard scale $\sim s$. In contrast to the handbag mechanism where there is only one active quark and antiquark, all quarks and antiquarks of the valence Fock components participate in the hard subprocess which necessitates the exchange of two hard gluons to lowest order of perturbative QCD. The baryons are described by ordinary three-quark distribution amplitudes, i.e. by wave functions integrated over quark transverse momenta. It turns out, however, that this factorization scheme fails badly with the normalization of the cross section [24], it is way below experiment, in particular if distribution amplitudes are used that are close to the asymptotic form which are favored by phenomenology [25], lattice QCD [26] and by light-cone sum rules $[27]^{4}$. Despite this apparent failure the perturbative QCD factorization scheme is presumably the correct mechanism for $s \sim-t \sim-u \rightarrow \infty$ while the handbag contribution dominates the process $\gamma \gamma \rightarrow B \bar{B}$ for large but not asymptotically large Mandelstam variables ${ }^{5}$. The perturbative QCD factorization scheme predicts an energy dependence of the integrated $B \bar{B}$ cross sections as $s^{-5}$ while, experimentally, the $p \bar{p}$ cross sections falls as $\simeq s^{-7.2}$. The energy dependence of the phenomenological annihilation form factors take that experimental result into account. Thus, our analysis is consistent with the dominance of the perturbative QCD factorization scheme at very large energies.

\section{$4 \quad p \bar{p} \rightarrow \gamma M$ phenomenology}

As reported in [11] the handbag approach works quite well for the $\gamma \pi^{0}$ channel as far as the energy and scattering-angle dependence is concerned. While the leading-order, collinear result (8) reproduces these features it fails with the normalization of the cross section by order of magnitude as is also the case for the space-like process, photoproduction of the $\pi^{0}$ meson off protons [18]. It is therefore suggestive to assume that the handbag factorization

\footnotetext{
${ }^{4}$ There is a variant of the perturbative QCD mechanism in which a baryon is viewed as being composed of a quark and a diquark [28]. This variant leads to much better agreement with experiment.

${ }^{5}$ As pointed out in [29] quark-hadron duality provides additional justification for the handbag approach in this kinematic domain - contributions from cat's ears topology where the two photons couple to different quarks, are likely suppressed.
} 
holds as well for other photon-meson channels with subprocesses evaluated to leading-order, collinear accuracy and a normalization that is sufficiently enhanced by a more general mechanism. An example of such a mechanism is the insertion of an infinite number of fermionic loops in the hard gluon propagator [30]. Such a mechanism likely leads to an enhancement of the normalization independent on the produced meson. In any case a channel independent-enhancement of the normalization will be assumed throughout this paper. In [11] the normalization has been fitted to the Fermilab data on $p \bar{p} \rightarrow \gamma \pi^{0}[31]$. Lack of data prevents a similar procedure for the $V_{L}$ channels.

In [11] the non-valence form factor has not been taken into account in the analysis. In this situation the $\eta$ and the $\eta^{\prime}$ meson are solely generated through the $\eta_{q}$ state. The inclusion of the non-valence form factor modifies the result for the ratio of the $\eta$ and $\eta^{\prime}$ cross sections presented in [11]; the physical mesons can also be created by the $s \bar{s}$ intermediate state. Neglecting the two-gluon Fock components of the $\eta$ and $\eta^{\prime}$ mesons, we find for the $\eta^{\prime}-\eta$ cross section ratio

$$
\frac{d \sigma / d t\left(p \bar{p} \rightarrow \gamma \eta^{\prime}\right)}{d \sigma / d t(p \bar{p} \rightarrow \gamma \eta)}=\tan ^{2} \Phi_{P}\left|\frac{1+\kappa_{P} \cot \Phi_{P}}{1-\kappa_{P} \tan \Phi_{P}}\right|^{2}
$$

where

$$
\kappa_{P}=\sqrt{2} \frac{f_{s}\langle 1 / \tau\rangle_{\eta_{s}}}{f_{q}\langle 1 / \tau\rangle_{\eta_{q}}} \frac{e_{s} \rho_{s}}{e_{u}+e_{d} \rho_{d}} .
$$

The ratio of the $\eta$ and $\eta^{\prime}$ cross section is independent on the scattering angle. This is also case for the $\eta-\pi^{0}$ ratio as can easily be checked. The decay constants, $f_{q}$ and $f_{s}$, for the basis states, $\eta_{q}$ and $\eta_{s}$, have been estimated in $[14,15]: f_{q}=1.07 f_{\pi}$ and $f_{s}=1.34 f_{\pi}$. The corresponding distribution amplitudes do not differ much otherwise large OZI rule violations would occur in conflict with experiment [32]. Taking all this information into account, we estimate $\kappa_{P}$ to amount to $\simeq 0.16 s_{0} / s$. The $\eta^{\prime}-\eta$ cross section ratio is shown in Fig. 3. For $s \rightarrow \infty$ it is simply given by $\tan ^{2} \Phi_{P}$. This is the result obtained in [11]. An analogous result is obtained for the $\eta / \pi^{0}$ ratio:

$$
\frac{d \sigma / d t(p \bar{p} \rightarrow \gamma \eta)}{d \sigma / d t\left(p \bar{p} \rightarrow \gamma \pi^{0}\right)}=\cos ^{2} \Phi_{P}\left[\frac{f_{q}\langle 1 / \tau\rangle_{\eta_{q}}}{f_{\pi}\langle 1 / \tau\rangle_{\pi}} \frac{e_{u}+e_{d} \rho_{d}}{e_{u}-e_{d} \rho_{d}}\right]^{2}\left|1-\kappa_{P} \tan \Phi_{P}\right|^{2}
$$

This ratio is clearly smaller than 1 . Assuming equal distribution amplitudes for the $\eta_{q}$ and the pion, we obtain a value of 0.17 for the $\eta / \pi^{0}$ ratio at $s$ about $10 \mathrm{GeV}^{2}$. This value increases slowly with $s$ up to a value of 0.22 . Such small 

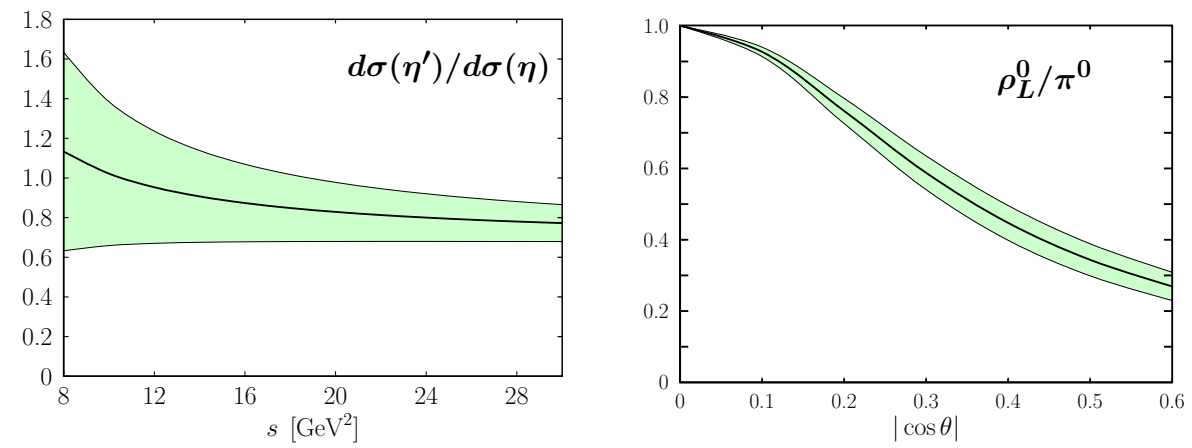

Figure 3: Left: The ratio of the $\eta^{\prime}$ and $\eta$ cross sections versus $s$. Right: The ratio of the $p \bar{p} \rightarrow \gamma \rho_{L}^{0}$ and $p \bar{p} \rightarrow \gamma \pi^{0}$ cross sections, scaled by their $90^{\circ}$ values, versus $|\cos \theta|$. For other notations refer to Fig. 2.

values of the $\eta / \pi^{0}$ ratio are also expected for deeply virtual kinematics [33] and have been observed experimentally [34].

The cross sections for the $\gamma V_{L}$ channels differ from that for the $\gamma \pi^{0}$ channel by the fact that $R_{\text {eff }}^{V_{L}}$ now goes along with the $\cos \theta$ dependence instead of $R_{V}^{V_{L}}$. As an example we show in Fig. 3 the angle dependence of the ratio of the $\gamma \rho_{L}^{0}$ and $\gamma \pi^{0}$ cross sections, scaled by the cross sections at $\theta=90^{\circ}$. The scaled ratio reads

$$
\frac{d \sigma / d t\left(p \bar{p} \rightarrow \gamma \rho_{L}^{0}\right)}{d \sigma / d t\left(p \bar{p} \rightarrow \gamma \pi^{0}\right)} / \frac{d \sigma / d t\left(p \bar{p} \rightarrow \gamma \rho_{L}^{0}, 90^{\circ}\right)}{d \sigma / d t\left(p \bar{p} \rightarrow \gamma \pi^{0}, 90^{\circ}\right)}=\frac{1+\cos ^{2} \theta\left(F_{\mathrm{eff}}^{u} /\left|F_{V}^{u}\right|\right)^{2}}{1+\cos ^{2} \theta\left(\left|F_{V}^{u}\right| / F_{\mathrm{eff}}^{u}\right)^{2}}
$$

It does not depend on energy.

Since the $\omega-\phi$ mixing angle is very small and the valence form factors are substantially larger than the non-valence one the strange quark admixture to the $\omega$ mesons can safely be ignored. Therefore, we find the simple result for the $\omega / \rho^{0}$ ratio

$$
\frac{d \sigma / d t\left(p \bar{p} \rightarrow \gamma \omega_{L}\right)}{d \sigma / d t\left(p \bar{p} \rightarrow \gamma \rho_{L}^{0}\right)} \simeq\left(\frac{f_{\omega}\langle 1 / \tau\rangle_{\omega}}{f_{\rho}\langle 1 / \tau\rangle_{\rho}}\right)^{2}\left|\frac{e_{u}+e_{d} \rho_{d}}{e_{u}-e_{d} \rho_{d}}\right|^{2}
$$

This is the analogue of (16) for a zero mixing angle. For the $\phi$ channel, on 


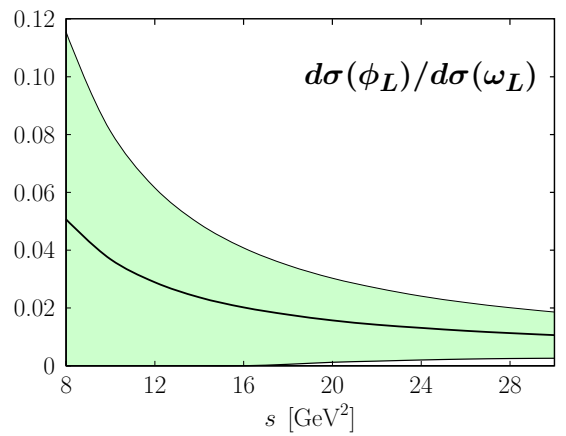

Figure 4: The ratio of the $\phi_{L}$ and $\omega_{L}$ cross sections versus $s$. For other notations refer to Fig. 2.

the other hand, we obtain ${ }^{6}$

$$
\frac{d \sigma / d t\left(p \bar{p} \rightarrow \gamma \phi_{L}\right)}{d \sigma / d t\left(p \bar{p} \rightarrow \gamma \omega_{L}\right)}=\tan ^{2} \Phi_{V}\left|1+\kappa_{V} \cot \Phi_{V}\right|^{2}
$$

where

$$
\kappa_{V}=\sqrt{2} \frac{f_{\phi}\langle 1 / \tau\rangle_{\phi}}{f_{\omega}\langle 1 / \tau\rangle_{\omega}} \frac{e_{s} \rho_{s}}{e_{u}+e_{d} \rho_{d}} .
$$

For $s \simeq s_{0}$ the strange quark content of the proton, embedded in the nonvalence form factor $F_{i}^{s}$, is strong enough to generate the $\phi$ meson through its $s \bar{s}$ Fock component. For large $s$, on the other hand, the non-valence form factor is suppressed and the $\phi$ is generated by the non-strange quarks through mixing which results in

$$
\frac{d \sigma / d t\left(p \bar{p} \rightarrow \gamma \phi_{L}\right)}{d \sigma / d t\left(p \bar{p} \rightarrow \gamma \omega_{L}\right)} \stackrel{s \rightarrow \infty}{\longrightarrow} \tan ^{2} \Phi_{V} .
$$

For an quantitative estimate of the vector meson cross sections we take $f_{\rho^{0}}=209 \mathrm{MeV}, f_{\omega}=187 \mathrm{MeV}$ and $f_{\phi}=221 \mathrm{MeV}$ [35] and the Gegenbauer coefficients of the $\rho^{0}$ and $\phi$ mesons from [36] $\left(a_{2}^{\rho}=0.2, a_{2}^{\phi}=0\right.$ at the scale of $\left.\mu_{0}=1 \mathrm{GeV}\right)$. For the $\omega$ meson the distribution amplitude is unknown

\footnotetext{
${ }^{6}$ Since the mixing angle is so small we approximate the decay constants of the nonstrange and strange Fock components of the $\omega$ and $\phi$ mesons by the phenomenological values of the physical mesons.
} 
and we assume that it equals that of the $\rho$ meson. The scale dependent $1 / \tau$ moment of a distribution amplitude is given by

$$
\langle 1 / \tau\rangle=3\left[1+a_{2}\left(\alpha_{\mathrm{s}}\left(\mu_{R}\right) / \alpha_{\mathrm{s}}\left(\mu_{0}\right)\right)^{50 / 81}\right] .
$$

For the renormalization (and factorization) scale we take the average gluon virtuality in the subprocess $\mu_{R}=\sqrt{s} / 2\left(\Lambda_{\mathrm{QCD}}=181 \mathrm{MeV}\right)$. Using these parameters, we estimate the $\omega / \rho^{0}$ ratio to amount to about $0.26 \pm 0.09$ independent on energy. The $\phi / \omega$ cross section ratio is displayed in Fig. 4.

The generalization of our approach to transversely polarized vector mesons is principally possible. In this case one has to consider the subprocess $q \bar{q} \rightarrow \gamma V_{T}$ with equal and opposite quark and antiquark helicities. In the first case this requires the introduction of new $p \bar{p}$ distributions (time-like versions of the transversity GPDs), and, hence, a new set of associated annihilation form factors. For opposite helicities higher-twist meson distributions are required. The analysis of $p \bar{p} \rightarrow \gamma V_{T}$ is beyond the scope of this work.

\section{Remarks on $J / \psi$ production}

One may also consider the process $p \bar{p} \rightarrow \gamma J / \psi$. The quark contribution to this process, i.e. the subprocess $c \bar{c} \rightarrow \gamma J / \psi$, is to be calculated analogously to the above discussed light-quark initiated processes except that the charm quark mass, $m_{c}$, is to be taken into account. The annihilation form factors $F_{i}^{c}$ are expected to be very small and to fall off with energy even more rapidly than $F_{i}^{s}$. Thus this contribution is likely very small as is the case for the space-like process, photoproduction of the $J / \psi$. As for the latter process (see e.g. [37]) there is also the possibility of the emission of a pair of gluons from the proton and antiproton which goes along with gluonic $p \bar{p}$ distribution amplitudes and associated gluonic annihilation form factors, $F_{i}^{g}(s)$. The structure of the $J / \psi$ cross section is similar to (6) with however different perturbative coefficients multiplying the gluonic annihilation form factors. In addition there is a contribution from configurations for which the gluons have the same helicity instead as opposite helicity. This contribution goes along with with a form factor being related to a time-like gluonic transversity GPD.

In principle the gluonic annihilation form factors can be determined from data on the $p \bar{p} \rightarrow \gamma J / \psi$ cross section in full analogy to the analysis of $\gamma \gamma \leftrightarrow$ $p \bar{p}$. With the gluonic form factors at hand it is possible to estimate the cross 
sections for reactions like $p \bar{p} \rightarrow \gamma \Upsilon$ or $p \bar{p} \rightarrow \gamma \Psi^{\prime}$. At FAIR such reactions can be measured. Finally we note that for such measurements large $-t$ and $-u$ are not required since the mass of the heavy quark provides a hard scale.

\section{Summary and Outlook}

We have investigated two classes of reactions, $\gamma \gamma \rightarrow B \bar{B}$ and $p \bar{p} \rightarrow \gamma M$, for wide-angle kinematics, i.e. at large Mandelstam variables $s,-t$ and $-u$. Within the handbag mechanism these reactions are complementary in so far as the soft physics information required for their description within a factorization approach, are encoded in the same set of annihilation form factors which represent lowest moments of $B \bar{B}$ distribution amplitudes, timelike versions of GPDs. In contrast to previous studies of these processes $[8,11]$ a non-valence form factor is taken into account in the numerical analysis. From the present $\gamma \gamma \rightarrow B \bar{B}$ data $[12,13]$ the annihilation form factors can be determined with the help of a few simplifying assumptions. The uncertainties of the form factors are however rather large. More and better data are required for an improvement. Using these form factors we are in the position to give a number of predictions for various $p \bar{p} \rightarrow \gamma M$ channels, in particular for longitudinally polarized vector mesons. These predictions can be probed at FAIR. This may lead to a better understanding of the wide-angle exclusive processes and to a more precise knowledge of the annihilation form factors, in particular to the role of strangeness in the proton for $z$ close to $1 / 2$ in the time-like region which corresponds to large $x$ in the space-like region. This seems to be important in view of the recent controversy about the strangeness content of the nucleon. Traditional PDF-fits assume $s_{p}(x)=$ $\bar{s}_{p}(x)$ to be substantially smaller than the light quark sea distribution with approximately the same $x$ dependence, while recent ATLAS data [38] suggest that there is no such suppression at small $x$. In addition earlier HERMES data [39] suggest that $s(x)$ is much smaller than usually assumed at large $x$. As the strange content of the proton enters $W^{ \pm}$-production cross sections at LHC this controversy has to be settled. We conclude that it would be good if measurements of $p \bar{p} \rightarrow \gamma M$ at wide-angle kinematics had a rather high priority within the FAIR program. 


\section{References}

[1] M. Meskauskas and D. Mueller, arXiv:1112.2597 [hep-ph].

[2] S. V. Goloskokov and P. Kroll, Eur. Phys. J. C 53, 367 (2008) [arXiv:0708.3569 [hep-ph]].

[3] M. Diehl, T. Gousset, B. Pire and O. Teryaev, Phys. Rev. Lett. 81, 1782 (1998) [hep-ph/9805380].

[4] B. Pire and L. Szymanowski, Phys. Lett. B 622, 83 (2005) [hep$\mathrm{ph} / 0504255]$.

[5] A. V. Radyushkin, Phys. Rev. D 58, 114008 (1998) [hep-ph/9803316].

[6] M. Diehl, T. Feldmann, R. Jakob and P. Kroll, Eur. Phys. J. C 8, 409 (1999) [hep-ph/9811253].

[7] M. Diehl, P. Kroll and C. Vogt, Phys. Lett. B 532, 99 (2002) [hep$\mathrm{ph} / 0112274]$.

[8] M. Diehl, P. Kroll and C. Vogt, Eur. Phys. J. C 26, 567 (2003), [hep$\mathrm{ph} / 0206288]$.

[9] A. Freund, A. V. Radyushkin, A. Schafer and C. Weiss, Phys. Rev. Lett. 90, 092001 (2003) [hep-ph/0208061].

[10] M. Diehl and P. Kroll, Phys. Lett. B 683, 165 (2010) [arXiv:0911.3317 [hep-ph]].

[11] P. Kroll and A. Schäfer, Eur. Phys. J. A 26, 89 (2005) [hep-ph/0505258].

[12] C. -C. Kuo et al. [Belle Collaboration], Phys. Lett. B 621, 41 (2005) [hep-ex/0503006].

[13] K. Abe et al. [Belle Collaboration], hep-ex/0609048.

[14] T. Feldmann, P. Kroll and B. Stech, Phys. Rev. D 58, 114006 (1998) [hep-ph/9802409].

[15] T. Feldmann, P. Kroll and B. Stech, Phys. Lett. B 449, 339 (1999) [hep-ph/9812269]. 
[16] J. Beringer et al. [Particle Data Group Collaboration], Phys. Rev. D 86, 010001 (2012).

[17] T. Feldmann and P. Kroll, Phys. Rev. D 62, 074006 (2000) [hep$\mathrm{ph} / 0003096]$.

[18] H. W. Huang and P. Kroll, Eur. Phys. J. C 17, 423 (2000) [hep$\mathrm{ph} / 0005318]$.

[19] M. Vanttinen and L. Mankiewicz, Phys. Lett. B 434, 141 (1998) [hep$\mathrm{ph} / 9805338]$.

[20] P. Achard et al. [L3 Collaboration], Phys. Lett. B 536, 24 (2002) [hepex/0204025].

[21] S. Anderson et al. [CLEO Collaboration], Phys. Rev. D 56, 2485 (1997) [hep-ex/9701013].

[22] G. P. Lepage and S. J. Brodsky, Phys. Rev. D 22, 2157 (1980).

[23] V. L. Chernyak and A. R. Zhitnitsky, Phys. Rept. 112, 173 (1984).

[24] G. R. Farrar, E. Maina and F. Neri, Nucl. Phys. B 259, 702 (1985) [Erratum-ibid. B 263, 746 (1986)].

[25] J. Bolz and P. Kroll, Z. Phys. A 356, 327 (1996) [hep-ph/9603289].

[26] V. M. Braun et al. [QCDSF Collaboration], Phys. Rev. D 79, 034504 (2009) [arXiv:0811.2712 [hep-lat]].

[27] I. V. Anikin, V. M. Braun and N. Offen, arXiv:1310.1375 [hep-ph].

[28] C. F. Berger and W. Schweiger, Eur. Phys. J. C 28, 249 (2003) [hep$\mathrm{ph} / 0212066]$.

[29] F. E. Close and Q. Zhao, Phys. Lett. B 553, 211 (2003) [hep$\mathrm{ph} / 0210277]$.

[30] A. V. Belitsky, AIP Conf. Proc. 698, 607 (2004) [hep-ph/0307256].

[31] T. A. Armstrong et al. [Fermilab E760 Collaboration], Phys. Rev. D 56, 2509 (1997). 
[32] P. Kroll and K. Passek-Kumericki, J. Phys. G 40, 075005 (2013) [arXiv:1206.4870 [hep-ph]].

[33] S. V. Goloskokov and P. Kroll, Eur. Phys. J. A 47, 112 (2011) [arXiv:1106.4897 [hep-ph]].

[34] V. Kubarovsky et al, Proceedings of the 4th Workshop " Exclusive reactions at High Momentum Transfer", Newport News, VA USA, 18-21 May 2010

[35] M. Beneke and M. Neubert, Nucl. Phys. B 675, 333 (2003) [hep$\mathrm{ph} / 0308039]$.

[36] P. Ball and V. M. Braun, hep-ph/9808229.

[37] D. Y. Ivanov, A. Schafer, L. Szymanowski and G. Krasnikov, Eur. Phys. J. C 34, 297 (2004) [hep-ph/0401131].

[38] G. Aad et al. [ATLAS Collaboration], Phys. Rev. Lett. 109, 012001 (2012) [arXiv:1203.4051 [hep-ex]].

[39] A. Airapetian et al. [HERMES Collaboration], Phys. Lett. B 666, 446 (2008) [arXiv:0803.2993 [hep-ex]]. Note that a substantial update of this analysis should be published in the near future by HERMES. 